\title{
The effect of medial septal and mammillary body lesions on the serial position curve in rats
}

\author{
DAVID N. HARPER, JOHN C. DALRYMPLE-ALFORD, and ANTHONY P. MCLEAN \\ University of Canterbury, Christchurch, New Zealand
}

\begin{abstract}
The effect of medial septum and mammillary body radio-frequency lesions on memory for list items was examined in rats. Subjects were exposed, one arm at a time, to a list of 7 arms presented in a 12-arm radial maze and were then required to return to a list arm in the subsequent test phase. Before surgery, rats in both groups displayed a serial position curve-that is, superior recognition for arms presented at the beginning of the list (primacy effect) and the end of the list (recency effect) but not the middle of the list. Medial septal lesions had two major effects on performance: accuracy at all serial positions was reduced, but while the primacy effect disappeared, a recency effect was retained. Following lesions of the mammillary bodies, the primacy and the recency effects disappeared, but there was no significant reduction in overall accuracy. The disruptive effect of both lesions on memory performance was robust despite extended training after surgery and was unaffected by increasing the exposure time to list arms during presentation. The memory impairment observed in rats following a medial septal lesion mimics the disruption in memory for list items that has been observed in patients with mild Alzheimer's disease and with Korsakoff's syndrome, two diseases that involve neuropathological changes in the medial septum.
\end{abstract}

In an important line of research in the neurosciences, the effect of specific brain lesions in rats has been examined in memory tasks that are analogous to those used with human subjects. An established procedure used with rats in this context is the serial probe recognition task, which allows the examination of memory for a list of spatial items (see Kesner, 1988a, 1988b). Humans and animals both show superior recognition for items at the beginning (called a primacy effect) and end (called a recency effect) of a list versus items from the middle of the list (see, e.g., Bolhuis \& van Kampen, 1988; Glanzer \& Cunitz, 1966; Harper, Dalrymple-Alford, \& McLean, 1992; Murdock, 1962; Wright, Santiago, Sands, Kendrick, \& Cook, 1985). However, the primacy effect is often reduced or eliminated in humans who suffer from the neurodegenerative disorders of Alzheimer's disease (AD) (Adelstein, Kesner, \& Strassberg, 1992; Gibson, 1981) and Korsakoff's syndrome (KS) (Baddeley \& Warrington, 1970). To help determine which aspects of the neuropathology present in these diseases play a role in the disruption of list memory, in the present study we investigated the effects of medial septum or mammillary body damage on a serial probe recognition task in rats.

An overlapping area of neuropathology in $\mathrm{AD}$ and $\mathrm{KS}$ is basal forebrain damage in the medial septum (Arendt, Bigil, Arendt, \& Tennstedt, 1983) and a subsequent loss of acetylcholine activity in the hippocampus (Antuono, Sorbi, Braco, Fusco, \& Amaducci, 1980). This overlap in neuropathology may underlie the behavioral overlap

Correspondence should be addressed to D. N. Harper, Department of Psychology, University of Otago, Dunedin, New Zealand (e-mail: harper@otago.ac.nz). observed between AD and KS in a number of memory tasks (see Delis et al., 1990). Of interest in the present study was whether the severe disruption in the primacy component of the serial position curve observed in both diseases could be modeled in rats by lesioning the medial septum. There is some evidence that primacy is disrupted more than recency by medial septal lesions in rats. For example, Kesner, Crutcher, and Beers (1988) examined the effect of medial septal lesions on memory for spatial list items. They presented rats with a list of five arms in an eight-arm radial maze. After a rat had visited each arm, its recognition of list arms was assessed by providing a choice between a previously seen list arm and a novel nonlist arm. Six rats with a relatively large medial septal lesion displayed an impairment in recognition accuracy at all serial positions in the list of five arms. Four other rats, which were found to have a relatively small medial septal lesion, showed no significant impairment at the final list position but were significantly impaired at all earlier positions. That is, rats with small medial septal lesions were similar to people with $\mathrm{AD}$ and $\mathrm{KS}$ in that performance on early and middle positions of the list was more impaired than performance on the final position(s).

However, the conclusions of Kesner et al.'s (1988) study are weakened by the observation that neither the control group nor the septal lesion group displayed clear primacy and recency effects prior to surgery. One possibile cause of the absence of clear serial position curves for some groups may have been the use of a $75 \%$ accuracy criterion (at both the first and the last serial positions) in baseline training. As noted by Gaffan (1992), such criteria do not allow an indication of the actual magnitude of primacy or recency effects, since the range of data vari- 
ability is restricted by the selection process. Another possible reason for the absence of clear serial position effects consists in the procedural limitations of using a 5-arm list in an 8-arm maze. When presented with a 5-arm list in an 8-arm maze, the rat can potentially reduce any demand on memory by remembering only the arms that have not been visited (a total of 3 arms at the end of a list presentation). However, with the use of a 12-arm maze instead of an 8-arm maze, the list length can be increased to 7 arms and still leave 5 arms unvisited for the comparison phase of a trial. Indeed, with the use of a 7-arm list presented in a 12-arm maze, robust and persistent primacy and recency effects do emerge (Harper et al., 1992). Therefore, we examined the effect of a small medial septal lesion in rats on the primacy and recency effects that emerge when a 7 -arm list is presented in a 12 -arm radial maze, to assess whether primacy would be reduced more than recency.

In addition to the effect of medial septal lesions, we also examined the effect of mammillary body lesions on primacy and recency effects in rats. The mammillary bodies receive input from the medial septum and hippocampus (Swanson, 1982), and like the medial septum, they are often found to be damaged in people with KS (Mair, Warrington, \& Weiskrantz, 1979; Mayes, Meudell, Mann, \& Pickering, 1988). Furthermore, damage in the mammillary bodies has been linked to the memory impairments observed in KS. For example, Mair et al. (1979) examined two cases of KS and noted a variety of memory deficits. At autopsy, the brains of both cases showed marked gliosis and shrinkage in the mammillary bodies, but no evidence of abnormality in the hippocampi, temporal lobes, or medial dorsal nucleus. The mammillary bodies have not, however, been implicated in the neuropathology or behavioral disruptions observed in AD. The case for claiming that the overlapping neuropathology in the medial septum is responsible for the overlapping behavioral disruptions in $K S$ and $A D$ would be strengthened by observing a different pattern of disruption following a mammillary body lesion as opposed to a medial septal lesion. Therefore, we examined the effect of mammillary body lesions on primacy and recency effects and compared the pattern of deficit to that observed with medial septal lesions.

A second issue addressed here is the robustness of changes in the serial position curve in both the medial septal and the mammillary body lesion groups. Since diseases such as AD and KS cause permanent memory disruptions, an ideal model of these diseases should produce robust deficits. However, it is not known how responsive the lesion effects are to extended training and changes in various aspects of the serial probe recognition task. Two means of assessing the robustness of the lesion effects were examined in the present study. The first consisted of training for an extended period of time after surgery. It may be that the observation of a memory disruption is limited to only a relatively restricted number of training trials before behavior recovers. In previous efforts to assess the effect of lesions on memory for list items, performance has only been examined over a small number of training trials. For instance, Kesner et al. (1988) examined performance over $\mathbf{4 0}$ trials (8 per serial position) following surgery, but the longer term aspects of performance were not assessed with training beyond this point. In the present study, we examined behavior over 120 trials (24 sessions) postlesion for medial-septallesioned rats and over 90 trials (18 sessions) postlesion for mammillary-body-lesioned rats. The second way in which the robustness of memory deficits was examined was to increase the time during which a rat was exposed to list arms during presentation. Research with humans has shown that increasing the exposure time to list items increases accuracy on items occurring at the beginning and middle of a list (Murdock, 1962). Therefore, extended exposure to list arms may aid the recognition of those arms, and thereby counteract any reductions in accuracy caused by the lesions, particularly at positions early in the list. For 6 sessions, medial-septal- and mammillarybody-lesioned rats were exposed to list arms for a longer period during presentation, to test whether lesion effects would be robust with extended exposure.

\section{METHOD}

\section{Subjects}

Twenty male Wistar rats approximately 14 months old at the start of the experiment and with extensive experience in the serial probe recognition task were used in the experiment. The rats had previously been used in the study of Harper et al., (1992), in which they received approximately 24 sessions (120 trials) of training in the same seven-arm list procedure that was used here. This previous study had indicated that the serial position curve was persistent and that the rats had reached assymptotic performance. The rats were housed 4 per cage and were maintained at $80 \%-85 \%$ of their freefeeding body weight with free access to water throughout the study.

\section{Apparatus}

The apparatus was an elevated $(85 \mathrm{~cm}$ from the floor) radial maze, comprising 12 evenly spaced aluminum arms radiating from a wooden central platform, $35 \mathrm{~cm}$ wide and painted black. Each arm was $9 \mathrm{~cm}$ wide and $65 \mathrm{~cm}$ long, with $3-\mathrm{cm}$ borders, a $25-\mathrm{cm}$-high clear plastic barrier at the proximal end, and a 2-cm-diam food well drilled in a wooden block placed at the distal end. Access to each arm was controlled via a clear plastic guillotine door located between the platform and the arm. Doors could be raised singly or in combination, by means of pulleys operated by the experimenter adjacent to the maze. The windowless room housing the maze was lit by two 22-W fluorescent lamps. A door, shelves, and table provided ample external cues to the maze, the position of which was kept constant.

\section{Procedure}

A serial probe recognition procedure was used to examine memory for list items. Each trial of training involved two phases-a presentation phase, in which a sequence of successively available arms was presented to the subject, followed by a test phase, in which recognition for one of the list arms was examined. At the start of each trial, a rat was placed on the central platform and all doors were lowered. A predetermined sequence of seven arms was then presented to the rat, one arm at a time. The door to the first arm in the sequence was raised, allowing the rat to run down the arm 
and gain a single piece of chocolate $(0.1 \mathrm{~g})$. When the rat returned to the central platform, the door was lowered and the door to the next arm in the list was raised. After all seven arms in the list sequence had been visited, one of the arms in List Position 1, 3, 4, 5 , or 7 was then rebaited with three pieces of chocolate before the experimenter simultaneously raised the door to the rebaited arm and the door to an unbaited arm that had not been visited in the previous list. The interval between the last door's being lowered in the presentation phase and the two doors' being raised in the test phase was $10 \mathrm{sec}$, and the rat was left in the apparatus during the delay. In the test phase, the rat was required to return to the one arm of the pair that it had visited during the presentation phase (i.e., match to sample). If the rat correctly returned to the list arm, it gained three pieces of chocolate; otherwise, it received nothing. Once three paws had entered an arm, the door was lowered and the rat was counted as having made a response. Six sequences of seven-arm lists were chosen that did not allow for a response strategy to aid recognition of the previously presented arm (e.g., the list $1,2,3,4,5,6,7$ was not allowed). Comparison arms (i.e., arms previously unvisited) chosen for each test phase of a trial were always next door but one to the correct arm. On $50 \%$ of the trials, the comparison arm was to the right of the correct arm, and on the other $50 \%$, it was to the left.

Sessions were run 5 days a week, and each daily session consisted of five trials per rat. Rats were exposed to only one sequence, repeated for all five trials in a given session. On each trial, the seven list arms were presented and recognition was then tested for an arm in Position 1, 3, 4, 5, or 7. To allow each of the five tested serial positions to be tested once (for each rat in a given session), on each trial a different list arm and one of the five nonlist arms were tested for recognition. The order of testing serial positions was varied across rats per session and within rats over sessions. After being run on a trial, the rat was removed from the apparatus and the next rat was run, until all rats had received one trial. The first rat was then run on the second trial of the session and so on until all the rats had completed their five trials for the day. Thus, successive trials for a given subject were normally at least $20 \mathrm{~min}$ apart. This current procedure has been shown to be successful in establishing clear and persistent serial position curves (see Harper et al., 1992), with rats not showing any systematic improvements or impairments in performance across the five trials in the session. Furthermore, apart from enabling the examination of each serial position in a single session, the current procedure overcomes many of the difficulties present in some manually based serial probe recognition procedures identified by Gaffan (1992). For instance, there was no selection criterion of the data included (cf. Kesner et al., 1988), which may have confounded the magnitude of primacy or recency effects. Also, arms were presented in different list positions, and equally often as the correct list item or as the novel comparison arm, thus reducing the possibility that a group preference for a certain arm (or arms) confounded accuracy at a given serial position (see Gaffan).

In order to maximize the use of the 20 subjects, 10 subjects served first as the sham-control group and then later as the mammillary body lesion group. Thus a single control group was used to control for both medial septal and mammillary body lesion effects. The current research began with examination of the effect of a small medial septal lesion on the serial position curve in rats. Training began with all 20 rats being run for six sessions ( 30 trials) in a baseline condition. Following completion of the baseline training, the rats were assigned to either a sham-operation control group ( $n=$ $10)$ or the medial septal lesion group $(n=10)$ in such a way that the serial position curves exhibited by each group prior to lesions were similar. The animals were allowed 5-7 days to recover following surgery, and then they were given the same behavioral procedure that had been used before surgery. Individuals from the two groups were tested in a mixed order. Following surgery, the rats in the medial septal group were tested for four blocks of six sessions per block ( 120 trials). The rats in the sham-operated control group were tested for three blocks of six sessions per block ( 90 trials) after surgery before receiving a mammillary body lesion. Since the sham surgery did not produce any observable cortical damage, the effects of mammillary body surgery were considered to be unconfounded by prior surgery. After being given a mammillary body lesion, the rats were allowed 5-7 days to recover and then they were given the same behavioral procedure that had been used before surgery. The 6 rats that survived mammillary body lesion surgery were trained for three blocks of six sessions per block (90 trials).

Following the fourth and third blocks of training after surgery for the medial-septal- and mammillary-body-lesioned groups, respectively, the effect of increasing the exposure time to list arms was examined. Exposure to list arms was increased by requiring the rat to spend a longer period of time down each arm during presentation, achieved by placing more chocolate at the end of a list arm. The procedure was the same as before, except that on every alternate session, three pieces of chocolate were placed at the end of each list arm in the presentation phase of a trial. On every other session, only one piece of chocolate was placed at the end of each list arm, as had been the case with previous baseline training. The rats were run for six sessions for each of these conditions. The mammillarybody-lesioned, but not the medial-septal-lesioned rats were then trained for six more sessions in the basic baseline procedure with only one piece of chocolate per arm in the presentation phase.

\section{Surgery}

Radio-frequency medial septal and mammillary body lesions were made with a Radionics RFG-4A lesion generator with a $0.25-\mathrm{mm}$ electrode lowered to the appropriate coordinates in the brain. The rats were anaesthetized with an i.p. injection of a xylazine $(50 \mathrm{mg} / \mathrm{kg}) / \mathrm{ketamine}(100 \mathrm{mg} / \mathrm{kg})$ mixture. The rat was then placed in a stereotaxic apparatus with the incisor bar set $3 \mathrm{~mm}$ below the interaural line. The medial septal lesion was made at the midline, $0.8 \mathrm{~mm}$ anterior to bregma and 5.0 and $5.8 \mathrm{~mm}$ below the dura. For the dorsal site, the temperature was raised to $60^{\circ} \mathrm{C}$ for $1 \mathrm{~min}$. For the ventral site, the temperature was raised to $65^{\circ} \mathrm{C}$ for $1 \mathrm{~min}$. For the sham-operated control group, the electrode was lowered into the cortex above the medial septum, but no current was passed. After a period of training, the sham group received a mammillary body lesion at $2.6 \mathrm{~mm}$ anterior to lambda, $\pm 0.5 \mathrm{~mm}$ lateral to the midline and $9 \mathrm{~mm}$ below the dura. At each site, the electrode temperature was raised to $65^{\circ} \mathrm{C}$ for $1 \mathrm{~min}$.

\section{Histology}

At the end of the experiment, all subjects were sacrificed and perfused, and their brains were removed for storage in $4 \%$ formaldehyde followed by a $30 \%$ sucrose-formalin solution. Two series of frozen coronal sections ( $40 \mu \mathrm{m}$ thick) were taken. One series was taken through the rostrocaudal extent of the medial septum. Every third section was mounted on a glass slide, which was then stained with cresyl violet. The size and location of the lesions were assessed by microscopic examination for the loss of neurons and the presence of gliosis. The other series of sections was obtained through the rostrocaudal extent of the hippocampus. Every third section was mounted on a glass slide, which was stained for the presence of acetylcholinesterase (AchE) to examine the extent of cholinergic denervation. The sections were incubated overnight in a solution containing cupric sulfate, glycine, ethoproprazine, and acetylthiocholine iodide and subsequently developed with sodium sulphide and enhanced with silver nitrate.

\section{RESULTS}

\section{Histology}

Reconstructions of the largest and smallest lesions in both the mammillary body and medial septal lesion groups 

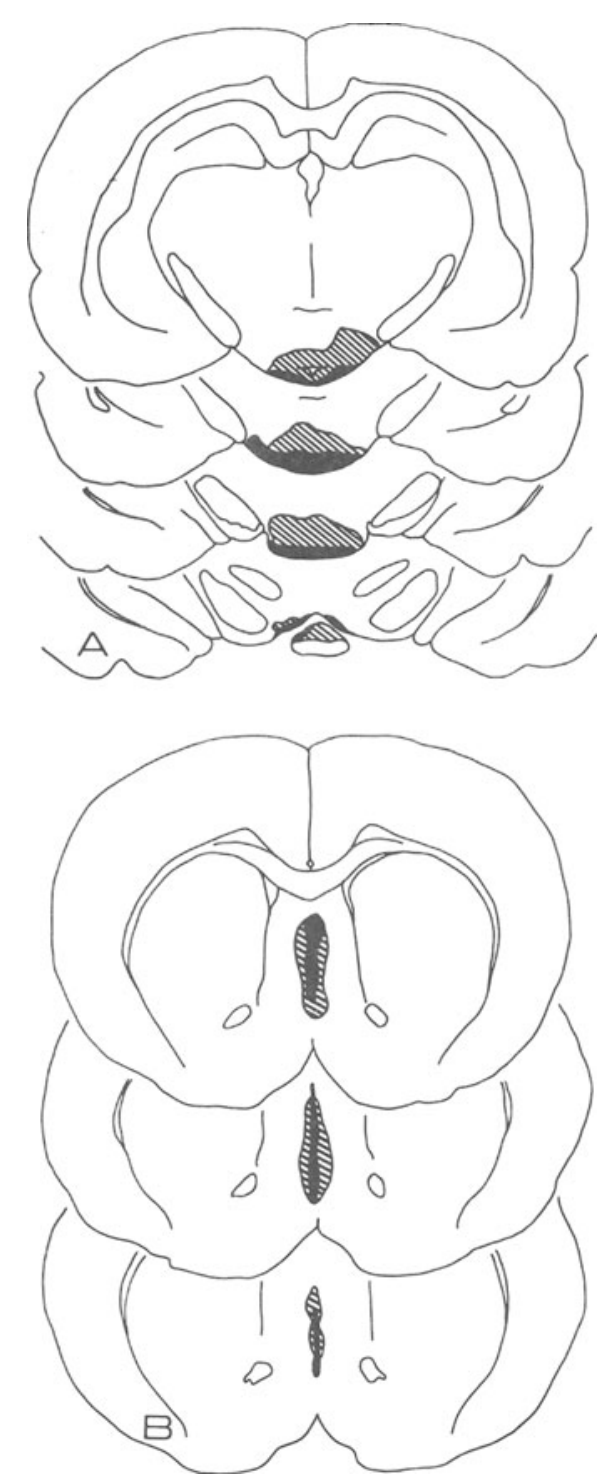

Figure 1. Schematic representation of the largest and smallest mammillary body lesion (A) and medial septal lesion (B) reconstructed from cresyl violet stained sections.

are shown in Figures $1 \mathrm{~A}$ and $1 \mathrm{~B}$, respectively. All 10 rats in the medial septal lesion group displayed appropriate damage to the medial septal area. All 6 rats in the mammillary body lesion group displayed damage to the mammillary body region. One rat in the mammillary body lesion group displayed damage located primarily in the lateral mammillary nuclei, but all rats displayed some damage to the posterior medial mammillary nucleus. The cholinergic denervation in the hippocampi of representative rats from the mammillary body and medial septal lesion groups is shown in Figures $2 \mathrm{~A}$ and $2 \mathrm{~B}$. The AchE sections for 1 medial-septal-lesioned rat were lost during processing. For the remaining 9 medial-septal-lesioned rats, there was a greater, although incomplete, bilateral reduction in AchE than there was for the 6 mammillary- body-lesioned rats. For 6 of the medial-septal-lesioned rats, the hippocampal AchE reduction was asymmetric. Although there was only a limited range of hippocampal AchE depletion, the greater the lesion size, the greater the denervation observed. However, the rats with the greatest depletion did not necessarily display the greatest behavioral changes (see the section below). For example, the rat with the greatest depletion displayed a change in serial probe recognition performance similar to that of the rat with the least depletion.

\section{Behavior}

The first issue addressed in the present research was the effect of medial septal and mammillary body lesions on the serial position curve in rats. The left panels in Figure 3 show the mean accuracy at Positions $1,3,4,5$, and 7 from the initial 6 sessions ( 30 trials) before surgery for the 10 medial-septal-lesioned rats, 6 mammillary-bodylesioned rats, and 10 sham-control rats. Note that because some members of the mammillary body lesion group were originally in the sham group, the baseline data for the mammillary body lesion group were obtained from the last 6 sessions of training following sham surgery. The right
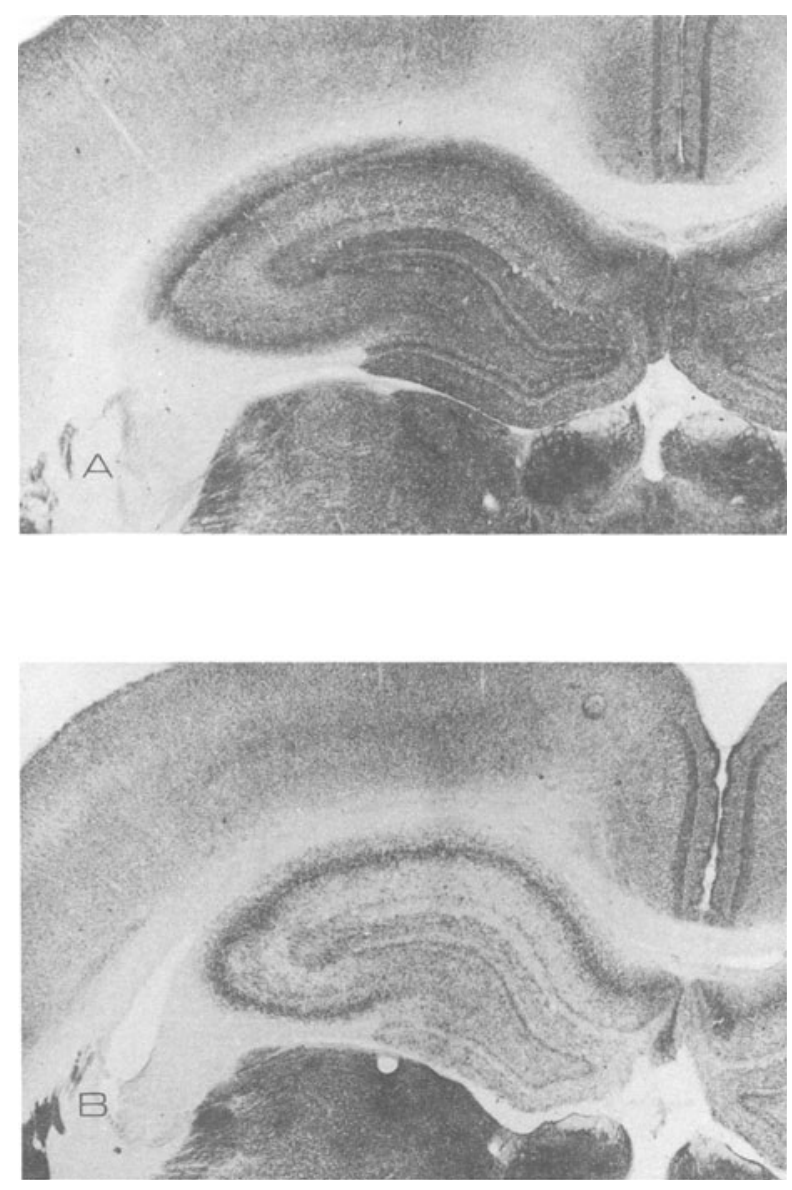

Figure 2. Degree of cholinergic innervation in the hippocampus as revealed by acetylcholinesterase histochemistry for a typical animal from the mammillary body (A) and medial septal (B) lesion group. 


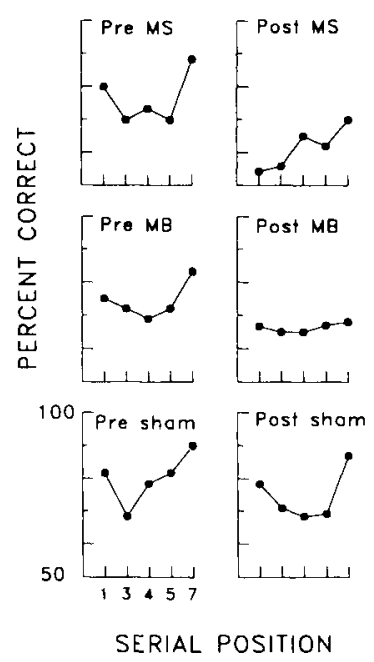

Figure 3. Percent correct recognition at Serial Positions 1, 3, 4, 5 , and 7 in a seven-arm list prior to (left column of graphs) and following (right column of graphs) surgery. The top, middle, and bottom rows of graphs show data from rats in the medial septal, mammillary body, and sham-control conditions, respectively. For each graph, the five points on the horizontal axis represent Serial Positions $1,3,4,5$, and 7 . The vertical axis gives percent correct, gained by averaging over all subjects in a group for a given serial position. For the presurgery graphs, the measure of percent correct was obtained from 6 sessions of training immediately prior to surgery. For the postsurgery graphs, the measure of percent correct was obtained from the 7th to 18th sessions of training following surgery.

panels in Figure 3 show the mean accuracy at Positions $1,3,4,5$, and 7 from Sessions 7-18 following medial septal, mammillary body, and sham surgery. Since the first 6 sessions of training after surgery revealed disruptive effects of surgery even for the control rats (see Figure 4), these data were considered to represent a period of refamiliarization with the task and were excluded from the data presented in Figure 3. Accuracy was measured by using the percentage of correct returns to a list arm during the test phase of a trial at a given serial position.

Figure 3 shows that medial septal, mammillary body, and sham surgery all had different effects on the serial position curve in rats. Before surgery, rats showed a serial position curve with primacy and recency effects present. That is, accuracy was higher at the two ends of the list than at the middle positions. The presence of a serial position curve was confirmed by the existence of a significant quadratic (i.e., U-shaped) trend in accuracy across serial positions for rats in the medial septal, mammillary body, and sham groups before surgery $[F(1,9)=6.05$, $p<.05 ; F(1,5)=6.67, p<.05$; and $F(1,9)=12.96$, $p<.01$, respectively]. Figure 3 also shows that after the 6-day recovery period following surgery, the rats in the sham-control condition continued to show a serial position curve with both primacy and recency effects present. Analyses for the sham-control group revealed no effect of surgery on performance $(F<1.0)$, and a significant quadratic trend in accuracy was still present $[F(1,9)=$ $31.12, p<.001]$.
Both medial septal and mammillary body lesion groups showed changes in recognition performance after surgery. The rats with medial septal lesions showed two major changes from their presurgery performance: overall accuracy decreased, and the primacy effect ceased to be evident. The decrease in accuracy over all serial positions was confirmed by a significant effect of surgery on performance $[F(1,9)=26.22, p<.001]$. A significant decrease in accuracy occurred at both Position $1[F(1,9)=$ 13.33, $p<.05]$ and Position $7[F(1,9)=18.47, p<$ $.01]$. However, despite a significant decrease in accuracy for Position 7, Figure 3 and the subsequent analyses demonstrated that a recency effect was maintained. Across serial positions, following surgery, accuracy was poorest for Position 1 and greatest for Position 7. Trend analysis revealed a significant positive linear component $[F(1,9)$ $=7.91, p<.05]$ in accuracy across serial positions but no evidence of a quadratic trend $(F<1.0)$ in the medial septal lesion group. Therefore, despite accuracy's decreasing overall, a recency effect was maintained due to accuracy's remaining superior for positions late in the list as opposed to positions early in the list. However, the primacy effect disappeared, because accuracy was no longer superior at early list positions as opposed to middle list positions.

The mammillary body lesion did not result in the changes in performance observed in the medial-septallesioned rats following surgery. The mammillary body lesion did not significantly decrease overall performance $(F<1.0)$, but it did eliminate evidence of the primacy and recency effects that had been present throughout extended training prior to mammillary body surgery. Therefore, despite the existence of a significant quadratic trend across serial positions before surgery, mammillary-bodylesioned rats failed to show the same trend following surgery. Furthermore, following the mammillary body lesion, the rats did not show the significant linear increase in accuracy across serial positions $(F<1.0)$ that was shown by the medial septal lesion group.

A second issue of interest in the present study was the robustness of medial septal or mammillary body lesion effects on memory for list arms. The robustness of lesion effects was assessed in two ways: first, by observing performance in the basic procedure over several sixsession blocks of training following surgery, and second, by examining whether postsurgery performance could be altered by increasing the time in which rats were exposed to list arms. The robustness of the lesion effects with extended training was examined by plotting performance in rats following medial septal, mammillary body, and sham surgery over a succession of six-session blocks, as shown in Figure 4. The top, middle, and bottom rows in Figure 4 show data following the medial septal, mammillary body, and sham lesions, respectively. The left panel in each row in Figure 4 shows accuracy at Positions 1, 3, 4,5 , and 7 in the first six sessions following surgery. The three subsequent panels (for the medial septal lesion group) and two subsequent panels (for the mammillary body lesion group and sham group) are successive 6-day 


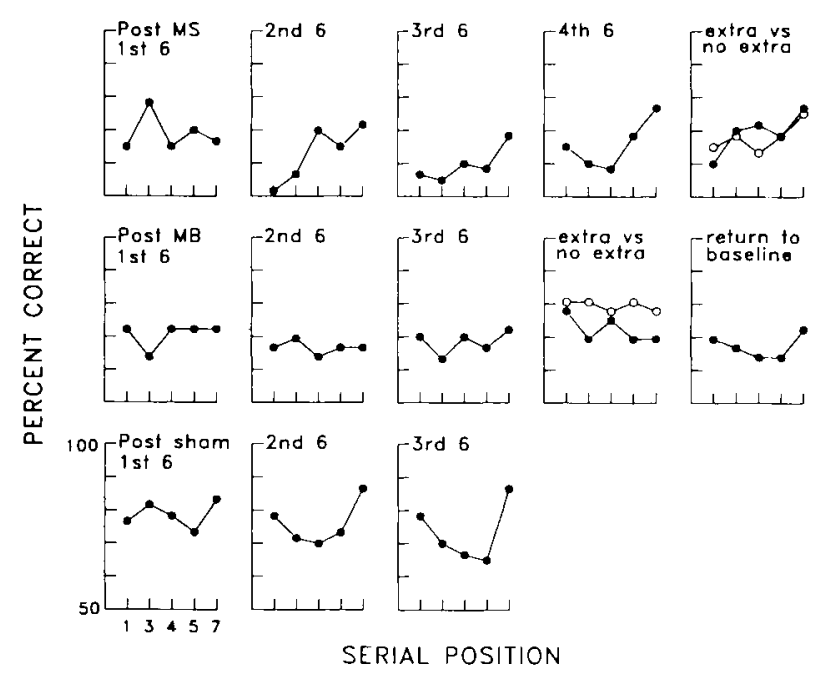

Figure 4. Percent correct at Serial Positions $1,3,4,5$, and 7 in a seven-arm list for medial-septal-lesioned, mammillary-bodylesioned, and sham-control rats broken down into sequential sixsession blocks of training following surgery. For each graph, the five points on the horizontal axis represent Serial Positions 1, 3, 4, 5 , and 7 , respectively. The vertical axis gives percent correct, gained by averaging over all subjects in a group for a given serial position across all six sessions shown in a graph. Also shown for the medialseptal- and mammillary-body-lesioned rats is a graph of accuracy across serial positions during the six sessions of extra-arm exposure (open circles) interpolated with six sessions of baseline with no extra exposure (closed circles).

blocks following surgery. Each data point represents the average percentage score from all the rats in a group over the six sessions of training in a block.

The first block of training following surgery shows that immediately after medial septal, mammillary body, and sham surgery, there was no evidence of a serial position curve. No significant trends in accuracy across serial positions were evident for any group. However, following the first postsurgery block, the trends summarized in Figure 3 were found to be robust across the subsequent sixsession blocks of training for all groups. Following sham lesions, the rats displayed a significant quadratic trend in accuracy across serial positions in Blocks $2[F(1,9)=$ $24.59, p<.001]$ and $3[F(1,9)=11.29, p<.01]$. Following medial septal lesions, the rats displayed a significant linear trend in accuracy across serial positions in Blocks $2[F(1,9)=13.95, p<.01], 3[F(1,9)=5.81$, $p<.05]$, and $4[F(1,9)=4.94, p<.05]$, but they failed to demonstrate a significant quadratic trend in any block. Also, the medial-septal-lesioned rats displayed a main effect of pre- versus postsurgery on accuracy in Blocks 2 $[F(1,9)=11.62, p<.01], 3[F(1,9)=34.09, p<$ $.001]$, and $4[F(1,9)=5.48, p<.05]$, indicating that accuracy was reduced across all serial positions for each block following surgery. Following mammillary body lesions, the rats failed to show any significant trends in ac- curacy across serial positions in any block or any significant reduction in accuracy overall.

The second means of examining the robustness of the lesion effects for the medial-septal-lesioned and mammillarybody-lesioned rats was to provide them with extended exposure to list arms during presentation. To extend the time spent down each arm during the presentation phase, the amount of chocolate placed at the end of each arm was extended from the baseline 1 piece to 3 pieces. This manipulation was effective in extending the period of time that rats spent down each arm in the list from an average of $53 \mathrm{sec}$ to an average of $82 \mathrm{sec}$ per rat in the medialseptal-lesioned group, and from an average of $51 \mathrm{sec}$ to one of $87 \mathrm{sec}$ per rat in the mammillary-body-lesioned group. The increase in time spent down list arms was not different for the two lesion groups (i.e., there was no interaction of group $\times$ condition, $F=1.23$ ). Across both groups, the increase in time spent down an arm due to the presence of extra chocolate was significantly greater than it was in the baseline condition $[F(1,14)=107.0$, $p<.001]$.

The effect of increased exposure to list arms on accuracy is shown in Figure 4 (see the fifth panel for medialseptal-lesioned rats and the fourth panel for mammillarybody-lesioned rats). These panels show performance during the six sessions of extra exposure to list arms (open circles) as opposed to the alternated six sessions with no extra exposure to list arms (filled circles). For the medialseptal-lesioned rats, the graph and subsequent analyses showed that despite an increase in exposure time to list arms, overall accuracy was not improved, nor was the positive linear trend in accuracy across serial positions altered. For the mammillary-body-lesioned rats, Figure 4 suggests that at Positions 3,5 , and 7 there was some increase in accuracy with extra list arm exposure in comparison with the baseline sessions. However, an analysis of the effect of extra exposure versus baseline on accuracy revealed no significant change in overall accuracy or accuracy at any given serial position. It may have been that the six sessions of extra exposure influenced accuracy during the interpolated six sessions of baseline in such a way that accuracy was improved during baseline, and hence an effect of condition could not be confirmed for the mammillary-body-lesioned rats. To check this possibility, a final six sessions of training under baseline conditions (see the fifth panel in the middle row of Figure 4) were conducted for the mammillary-body-lesioned rats, to observe whether accuracy in the baseline declined when training was not interpolated with the extra-exposure condition. However, there was no significant difference in accuracy between this baseline conducted without interpolated training and the six sessions of extra-arm exposure. Therefore, despite spending a significantly longer time down each list arm when extra chocolate was available, both groups failed to show any reliable change in accuracy in the present task. 


\section{DISCUSSION}

In the present study, both the medial septal and the mammillary body lesions resulted in a disruption in memory for list items. The medial septal lesion resulted in a decrease in accuracy at all serial positions of a 7-arm list presented in a 12-arm maze. Although overall accuracy was reduced, a recency effect reemerged after 30 trials of postlesion training and remained throughout the rest of the experiment, an additional 150 trials per rat. The mammillary body lesion group showed a different pattern of impairment from that of the medial septal lesion group. The mammillary body lesion did not reduce overall accuracy but did remove the primacy and recency effects. During subsequent training, the serial position curve did not reemerge for the mammillary body lesion group, and accuracy did not vary as a function of serial position. Therefore, the effect of lesions differed between the two groups and resulted in memory deficits that were robust with continued training.

The effect of surgery in the medial-septal- and mammillary-body-lesioned rats was robust even when the rats spent a significantly longer time at the end of each list arm, a procedure intended to improve performance at the middle and early list positions in the recognition task. One potential problem of increasing the exposure to each list arm was that the time between presentation of a list arm and subsequent recognition was also greater than it was in sessions without extra exposure, and this increase in delay was greater for earlier list positions than later ones. Harper, McLean, and Dalrymple-Alford (in press) have shown that with an increase in delay of 30-60 sec between presentation of a list and subsequent recognition, the primacy effect is reduced. Hence, any possible improvements in early list positions may have been restricted under the present procedure, and this may explain why the medial-septal- and mammillary-bodylesioned groups did not show a restoration of the primacy effect. However, it is unlikely that potential improvements at early list positions were inhibited in this way. In both lesion groups, it was not just memory for early serial positions that failed to increase significantly but also accuracy at the middle serial positions. Also, previous research with humans has shown that accuracy at both the early and middle serial positions increased with increased exposure time to list items despite simultaneously increasing the delay between presentation of these list items and subsequent testing (e.g., Murdock, 1962).

The effect of the medial septal lesion on memory for list items in the 10 rats in the present study is similar to that observed by Kesner et al. (1988). In 4 rats with small medial septal lesions, they found that accuracy at early positions in a five-arm list was reduced and that the primacy effect was thereby not evident following surgery, although a recency effect was present. However, in Kesner et al.'s study, primacy and recency effects were not clearly evident prior to surgery. The present findings indicated that even when there were clear primacy and recency effects before surgery, there was a differential effect of medial septal lesions on primacy versus recency. The present study not only adds support to Kesner et al.'s observations, but also extends them by showing that the effect of small medial septal lesions is robust with continued training and with extra exposure to list arms.

Interpreting the differential effects of the medial septal and mammillary body lesions on the serial position curve at the behavioral level is difficult, because of the variety of theoretical interpretations that have been suggested to account for primacy and recency effects. Some theorists attribute primacy and recency effects to differential processing within long- or short-term memory (see, e.g., Atkinson \& Shiffrin, 1968; Craik, 1970; Glanzer, 1972). Others have accounted for primacy and recency in terms of the action of retroactive and proactive interference (e.g., Postman \& Phillips, 1965; Waugh, 1960) or in terms of the delay reduction in reinforcement properties of stimuli that occur in different serial positions (e.g., Wixted, 1989). However, none of the theories have been able to account for all the data available on the serial position effect (Baddeley, 1986; Koppenaal \& Glanzer, 1990). Therefore, to interpret the effects of lesions on list-item memory in terms of a single model of memory is problematic.

An analysis of alterations in accuracy as a product of trial in the session suggested that the general decrease in accuracy observed at all serial positions for the medial septal lesion group was due to a buildup of proactive interference across trials. An analysis of accuracy at Positions 1,4 , and 7 when each position was tested in the first, middle, or last trial of the session indicated that for all groups before surgery and for all groups after surgery (except the medial septal lesion group), there were no significant alterations in accuracy at a given serial position or across all serial positions overall. This lack of influence of previous trials on later ones was the same as that observed in previous research done with the present procedure (Harper et al., 1992). However, for the medial septal lesion group in the 12 sessions following surgery, there was a significant reduction in accuracy on later trials in the session as opposed to early trials in the session $[F(2,18)=7.08, p<.05]$. Specifically, for the medial septal lesion group, following surgery, accuracy (across Positions 1, 4, and 7) was on the average $69 \%$ for the first trial of the session, $62 \%$ for the third, and $54 \%$ for the fifth. This trend was consistent with the possibility that medial septal damage may result in a greater susceptibility to proactive interference effects from previous trials. However, there was no significant interaction of serial position $\times$ trial of the session. Therefore, although medial septal lesions may result in a sensitivity to proactive interference effects from previous trials, and thus in an impairment in performance with continued testing in the session, this did not account for the differential influence of medial septal damage on the primacy and recency effects.

At the neurological level, it has been suggested that medial septal damage affects primacy because of the dis- 
ruption of acetylcholine activity in the hippocampal formation (Kesner et al., 1988). This view is supported by the observation that lesions of the dorsal hippocampal formation in rats (Kesner \& Adelstein, 1989; Kesner et al., 1988) and that humans with hippocampal damage (Milner, 1972) display patterns of list memory disruption similar to those in rats with medial septal lesions. However, because research to date has investigated electrolytic lesions (e.g., Kesner et al., 1988) or radio-frequency lesions (the present study) of the medial septum, it remains to be established that disruption in hippocampal acetylcholine activity rather than nonspecific fibre or cell damage is responsible for list-item memory impairment. Indeed, in the present study, although there was only a limited range of AchE depletion across the medial septal lesion group, the level of denervation did not reflect the amount of behavioral disruption exhibited by an animal.

Although the mammillary body region receives input from the medial septum and hippocampus (Swanson, 1982), the mammillary body region does not appear to serve the same role as the medial septum or hippocampus in memory for list items. For instance, whereas the mammillary body lesion removed the primacy effect, the removal of recency as well suggests that the medial septal and mammillary body regions are involved in different aspects of memory functioning. Furthermore, the lack of overall reduction in accuracy following the mammillary body lesion suggests that damage in the mammillary body region is not as disruptive to list-item memory as damage in the medial septum or hippocampus. There are, however, similarities between the effects of mammillary body lesions (in the present study) and nucleus basalis magnocellularis (NBM) lesions (observed in other studies) on memory for list items. For example, Kesner and Adelstein (1989) found that although small and large NBM lesions did not reduce overall performance, there was no evidence of primacy or recency effects following surgery for the large NBM group. In general, despite a number of studies of the effect of mammillary body lesions in a variety of tasks, the role of the mammillary body region in memory remains equivocal. The lack of overall reduction in memory performance on the serial probe recognition task following a mammillary body lesion is consistent with previous research, which has shown no or only limited impairment due to mammillary body lesions in delayed nonmatching-to-sample tasks (e.g., Aggleton, Hunt, \& Shaw, 1990; Aggleton, Keith, \& Sahgal, 1991) and an eight-arm radial maze task (Jarrard, Okaichi, Steward, \& Goldschmidt, 1984). However, other studies have reported a significant impairment in performance following mammillary body lesions in delayed nonmatchingto-sample and the eight-arm radial maze task (Saravis, Sziklas, \& Petrides, 1990), and in alternation tasks (Béracochéa \& Jaffard, 1987; Field, Rosenstock, King, \& Greene, 1978).

Of the two types of lesions investigated in the present study, only the medial septal lesion appears to mimic the list-item memory deficits observed in both $\mathrm{AD}$ and $\mathrm{KS}$. The medial septal lesion not only damaged cells in the medial septal region but also decreased the presence of acetylcholine activity in the hippocampus. These aspects of the neurological damage occur in both AD (Nakano \& Hirano, 1982; Vogels et al., 1990) and KS (Antuono et al., 1980; Arendt et al., 1983). Behaviorally, the medial septal lesion resulted in a severe impairment in memory for early list arms, but less of an impairment for later list arms, as observed in mild AD and KS (Adelstein et al., 1992; Baddeley \& Warrington, 1970). Furthermore, the finding that accuracy was generally reduced as training proceded through a session is consistent with the suggestion that medial-septal-lesioned rats displayed sensitivity to the disruptive influence of previous trials on later ones. Sensitivity to the potentially disruptive influence of previous list learning (albeit with lists containing different stimulus items) is also a feature of list recall in $\mathrm{AD}$ and KS. For example, Kramer et al. (1988) and Delis et al. (1991) have demonstrated that AD and KS subjects show a tendency to recall previous list items when asked to recall a more recent list.

Not only do rats with a medial septal lesion mirror the basic pattern of memory disruption observed, they also mirror the lack of improvement shown by AD sufferers under procedures that promote an increase in accuracy. For example, Miller (1971) showed that recall improved at early and middle positions for control subjects when the rate of stimulus presentation of list items was decreased. In contrast, for demented subjects, the low recall shown at early and middle list items was unaltered when the rate of stimulus presentation was decreased. Similarly, in the present study, increasing the amount of exposure to list items, which has been shown in humans to improve accuracy at early and middle list positions (Murdock, 1962), did not improve the performance of medial-septal-lesioned rats. Therefore, the present results are consistent with the suggestion that the similar neuropathology of the medial septum observed in both AD and KS may underlie the overlapping patterns of memory deficits for list items. However, it should be noted that when the stimuli used are of the "order" of list arms, the medial septal region does not appear to result in the memory impairments observed in AD (Kesner, 1988a). Therefore, the ability of any specific brain damage to result in a behavioral change that mimics a neurological disorder may be restricted to only one or a few behavioral tasks, and a clear picture of the functional importance of areas such as the medial septum and mammillary bodies will only emerge after a variety of experimental paradigms have been explored.

\section{REFERENCES}

Adelstein, T. B., Kesner, R. P., \& Strassberg, D. S. (1992). Spatial recognition and spatial order memory in patients with dementia of the Alzheimer's type. Neuropsychologia, 30, 59-67.

Aggleton, J. P., Hunt, P. R., \& Shaw, C. (1990). The effects of mammillary body and combined amygdalar-fornix lesions on tests of delayed non-matching to sample in the rat. Behavioural Brain Research, 40, 145-157.

Aggleton, J. P., Keith, A. B., \& Sahgal, A. (1991). Both fornix and anterior thalamic, but not mammillary, lesions disrupt delayed 
non-matching to position memory in rats. Behavioural Brain Research, 44, 151-161.

antuono, P., Sorbi, S., Braco, L., Fusco, T., \& Amaducci, L. (1980). A discrete sampling technique in senile dementia of the Alzheimer type and alcoholic dementia: Study of the cholinergic system. In L. Amaducci, A. N. Davison, \& P. Antuono (Eds.), Aging of the brain and dementia (pp. 151-158). New York: Raven Press.

Arendt, T., Bigil, V., Arendt, A., \& Tennstedt, A. (1983). Loss of neurons in the nucleus basalis of Meynert in Alzheimer's Disease, paralysis agitans and Korsakoff's disease. Acta Neuropathologia, 61, 101-108.

AtKinson, R. C., \& SHIFfrin, R. M. (1968). Human memory: A proposed system and its control processes. In K. W. Spence \& J. T. Spence (Eds.), The psychology of learning and motivation: Advances in research and theory (Vol. 1, pp. 89-195). San Diego, CA: Academic Press.

Baddeley, A. D. (1986). Working memory. Oxford: Oxford University Press.

Baddeley, A. D., \& Warkington, E. K. (1970). Amnesia and the distinction between long-and short-term memory. Journal of Verbal Learning \& Behavior, 9, 176-189.

BÉrACOCHÉA, D. J., \& JAFFARD, R. (1987). Impairment of spontaneous alternation behavior in sequential test procedures following mammillary body lesions in mice: Evidence for time-dependent interferencerelated memory deficits. Behavioral Neuroscience, 101, 187-197.

Bolmuis, J. J., \& Van Kampen, H. S. (1988). Serial position curves in spatial memory of rats: Primacy and recency effects. Quarterly Journal of Experimental Psychology, 40B, 135-149.

CraIK, F. I. M. (1970). The fate of primary memory items in free recall. Journal of Verbal Learning \& Verbal Behavior, 9, 143-148.

Delis, D. C., Massman, P. J., Butters, N., Salmon, D. P., Cermak, L. S., \& Kramer, J. H. (1991). Profiles of demented and amnesic patients on the California Verbal Learning Test: Implications for the assesment of memory disorders. Psychological Assessment: A Joumal of Consulting \& Clinical Psychology, 3, 19-26.

Field, T. D., Rosenstock, J., King, E. C., \& Greene, E. (1978). Behavioral role of the mammillary efferent system. Brain Research Bulletin, 3, 451-456.

GafFan, E. A. (1992). Primacy, recency, and the variability of data in studies of animals' working memory. Animal Learning \& Behavior, 20, 240-252.

GiBSON, A. J. (1981). A further analysis of memory loss in dementia and depression in the elderly. British Journal of Clinical Psychology, 20, 179-185.

Glanzer, M. (1972). Storage mechanisms in recall. In G. H. Bower (Ed.), The psychology of learning and motivation: Advances in research and theory (Vol. 5, pp. 129-193). New York: Academic Press.

Glanzer, M., \& CUnitz, A. R. (1966). Two storage mechanisms in free recall. Journal of Verbal Leaming \& Verbal Behavior, 5, 351-360.

Harper, D. N., Dalrymple-Alford, J. C., \& McLean, A. P. (1992). Production of a serial position effect in rats using a 12 -arm radial maze. Journal of Neuroscience Methods, 44, 197-207.

Harper, D. N., Mclean, A. P., \& Dalrymple-Alford, J. C. (in press). List item memory in rats: Effects of delay and delay task. Journal of Experimental Psychology: Animal Behavior Processes.

Jarrard, O. E., Okaichi, H., Steward, O., \& Goldschmidt, R. (1984). On the role of hippocampal connections in the performance of place and cue tasks: Comparisons with damage to the hippocampus. Behavioral Neuroscience, 98, 946-954.
KESNER, R. P. (1988a). Parallels between animals and humans in terms of the neural foundations of memory: Implications for Alzheimer's disease. In H. J. Altman \& B. N. Altman (Eds.), National Conference on Alzheimer's Disease and Dementia: Problems, prospects and perspectives (pp. 209-220). New York: Plenum.

KESNER, R. P. (1988b). Reevaluation of the contribution of the basal forebrain cholinergic system to memory. Neurobiology of Aging, 9 , 609-616.

Kesner, R. P., \& Adelstein, T. B. (1989). Equivalent spatial location memory deficits in rats with medial septum or hippocampal formation lesions and patients with dementia of the Alzheimer's type. Brain \& Cognition, 9, 289-300.

Kesner, R. P., Crutcher, K. \& Beers, D. R. (1988). Serial position curves for item (spatial location) information: Role of the dorsal hippocampal formation and medial septum. Brain Research, 454, 219-226.

Kramer, J. H., Delis, D. C., Blusewicz, M. J., Brandt, J., Ober, B. A., \& Strauss, M. (1988). Verbal memory errors in Alzheimer's and Huntington's dementias. Developmental Neuropsychology, 4, 1-15.

Mair, W. G. P., Warrington, E. K., \& Weiskrantz, L. (1979). Memory disorder of Korsakoff's psychosis: A neuropathological and neuropsychological investigation of two cases. Brain, 102, 749-783.

Mayes, A. R., Meudell, P. R., ManN, D., \& Pickering, A. (1988) Location of lesions in Korsakoff's syndrome: Neuropsychological and neuropathological data on two patients. Cortex, 24, 367-388.

MILLER, E. (1971). On the nature of the memory disorder in presenile dementia. Neuropsychologia, 9, 75-81.

MilneR, B. (1972). Disorders of learning and memory after temporal lobe lesions in man. Clinical Neurosurgery, 19, 421-446.

Murdock, B. B. (1962). The serial position effect in free recall. Journal of Experimental Psychology, 64, 482-488.

NaKano, I., \& Hirano, A. (1982). Loss of large neurons in the medial septal nucleus in an autopsy case of Alzheimer's disease. Journal of Neuropathology \& Experimental Neurology, 41, 431.

Postman, L., \& Phillips, L. W. (1965). Short-term temporal changes in free recall. Quarterly Joumal of Experimental Psychology, 17, $132-138$

Saravis, S., Sziklas, V., \& Petrides, M. (1990). Memory for places and the region of the mammillary bodies. European Joumal of Neuroscience, 2, 556-564.

SWANSON, L. W. (1982). Normal hippocampal circuitry: Anatomy. In L. W. Swanson, T. J. Teylor, \& R. F. Thompson (Eds.), Hippocampal long-term potentiation: Mechanisms and implications for memory. Neurosciences Research Program Bulletin, 20, 624-634.

Vogels, O. J. M., Broere, C. A. J., Ter laAk, H. J., Ten Donkelaar, H. J., NieuwenhuYs, R., \& SChulte, B. P. M. (1990). Cell loss and shrinkage in the nucleus basalis Meynert complex in Alzheimer's disease. Neurobiology of Aging, 11, 3-13.

WaUGH, N. C. (1960). Serial position and the memory span. American Journal of Psychology, 73, 68-79.

WiXTED, J. T. (1989). Nonhuman short-term memory: A quantitative reanalysis of selected findings. Journal of the Experimental Analysis of Behavior, 52, 409-426.

Wright, W. A., Santiago, H. C., Sands, S. F., Kendrick, D. F., \& CooK, R. G. (1985). Memory processing of serial lists by pigeons, monkeys and people. Science, 229, 287-289.

(Manuscript received September 16, 1992; revision accepted for publication December 17, 1992.) 